\title{
mRNA Dependent Virtual-Real Substitutions of Nucleotides in Codons: The Dynamics of Their Meanings in the Genome Language
}

\author{
P. P. Gariaev, E. A. Leonova-Gariaeva \\ Institute of Quantum Genetics LLC, Moscow, Russia \\ Email: gariaev@mail.ru
}

How to cite this paper: Gariaev, P.P. and Leonova-Gariaeva, E.A. (2019) mRNA Dependent Virtual-Real Substitutions of Nucleotides in Codons: The Dynamics of Their Meanings in the Genome Language. Open Journal of Genetics, 9, 77-90.

https://doi.org/10.4236/ojgen.2019.94006

Received: October 3, 2019

Accepted: December 2, 2019

Published: December 5, 2019

Copyright $\odot 2019$ by author(s) and Scientific Research Publishing Inc. This work is licensed under the Creative Commons Attribution International License (CC BY 4.0).

http://creativecommons.org/licenses/by/4.0/

\begin{abstract}
This is an attempt to explain mRNA-dependent non-stationary semantic values of codons (triplets) and nucleotides (letters) in codon composition during protein biosynthesis. This explanation is realized by comparing the different protein codes of various biosystem taxa, and, comparing mitochondrial code with the standard code. An initial mRNA transcriptional virtuality (VirtualReality) is transformed into material reality at the level of translation of virtual triplets into real (material) amino acids or into a real stop command of protein biosynthesis. The transformation of virtuality into reality occurs de facto when the linguistic sign ${ }^{1}$ functions of the codon syhoms are realized in the 3 ' nucleotide (wobbling nucleotide according to F. Crick) in the process of protein biosynthesis. This corresponds to the theoretical works of the authors of this article. Despite the illusory appearance of semantic arbitrariness during the operation of ribosomes in the mode of codon semantic non-stationarity, this phenomenon probably provides biosystems with an unusually high level of adaptability to changes in the external environment as well as to internal (mental) dynamics of neuron's genome in the cerebral cortex. The genome's non-stationarity properties at the nucleotide, codon, gene and mental levels have fractal structure and corresponding dimensions. The highest form of such fractality (with maximum dimension) is probably realized in the genomic continuum of neurons in the human cerebral cortex through this semantic Virtual-to-Real (VR) codon transcoding with the biosynthesis of short-living semantic proteins, as the equivalents of material
\end{abstract}


thinking-consciousness. In fact, this is the language of the brain's genome, that is, our own language. In this case, the same thing happens in natural, primarily mental (non-verbal) languages. Their materialization is recorded in vocables (sounding words) and in writing. Such writing is the amino acid sequence in the semantic proteins of the human cerebral cortex. Rapidly decaying, such proteins can leave a long-lasting "so-called" Schrödinger wave holographic memory in the cerebral cortex. The presented below study is purely theoretical and based on a logical approach. The topic of the study is very complex and is subject to further development.

\section{Keywords}

Protein Biosynthesis, Protein Codes, Codons (Triplets), mRNA, Virtual-Real (VR) Transcoding, Semantics, Thinking, Consciousness

\section{Introduction}

Let's consider a structurally modified standard (Table 1) of the Genetic Protein Code. The table presents symmetrically distributed codons: synonyms and syhoms (hybrids of synonymous codons and homonymous codons). The amended table was proposed by us earlier [1] [2].

A careful analysis of the table shows that the synonymous codon family TCT TCC TCA TCG encodes serine. Two triplets of syhom codons AGT AGC also encode serine. This represents a real material transformation of the coding doublets AG ---> TC, which enables syhom AG doublet to encode serine along the synonymous path.

Synonymous codon family CGT CGC CGA CGG encodes arginine. Two triplets of syhom codons AGA AGG also encode arginine. This represents a real material transformation of the coding doublets AG ---> CG, which enables the syhom AG doublet to encode arginine along the synonymous path.

Synonymous codon family CTT CTC CAA CTG encodes leucine. Two triplets of syhom codons TTA TTG also encode leucine. This represents a real material transformation of the coding doublets TT ---> CT, which enables syhom TT doublet to encode leucine on the synonymous path.

The observed phenomena can be the cause and effect of VR-transcoding of mRNA, and, can be manifested in codon frequency shifts (CFS) or codon usage bias. For example, on mRNA one of the synonymous codons of TC family can be "read" depending on mRNA context: it will be read not as encoding serine, but virtually transcoded as part of the AG syhom family. And consequently, during the translation, the transformation of virtuality into reality (VR-transcoding) will take place: and arginine (not serine) will be included into the synthesized protein due to VR-transcoding of TC $\rightarrow$ AG families. In sequencing of the resulting protein, we will discover substitution of serine by arginine. This will be the evidence of TC $\rightarrow$ AG family VR-transcoding during translation. 
Table 1. The table of the genetic (protein) code.

\begin{tabular}{|c|c|c|c|c|c|c|c|c|}
\hline & \multicolumn{2}{|c|}{ C } & \multicolumn{2}{|c|}{ G } & \multicolumn{2}{|c|}{$\mathrm{T}(\mathrm{U})$} & \multicolumn{2}{|c|}{ A } \\
\hline \multirow{4}{*}{$\mathrm{T}(\mathrm{U})$} & TCT & Ser & TGT & Cys & TTT & Phe & TAT & Tyr \\
\hline & TCC & Ser & TGC & Cys & TTC & Phe & TAC & Tyr \\
\hline & TCA & Ser & TGA & Stop & TTA & Leu & TAA & Stop \\
\hline & TCG & Ser & TGG & $\operatorname{Trp}$ & TTG & Leu & TAG & Stop \\
\hline \multirow{4}{*}{ A } & ACT & Thr & AGT & Ser & ATT & Ile & AAT & Asn \\
\hline & ACC & Thr & AGC & Ser & ATC & Ile & AAC & Asn \\
\hline & $\mathrm{ACA}$ & The & AGA & Arg & ATA & Ile & $\mathrm{AAA}$ & Lys \\
\hline & ACG & Thr & AGG & Arg & ATG & Met & AAG & Lys \\
\hline \multirow{4}{*}{ C } & $\mathrm{CCT}$ & Pro & CGT & Arg & CTT & Leu & CAT & His \\
\hline & $\mathrm{CCC}$ & Pro & CGC & Arg & CTC & Leu & $\mathrm{CAC}$ & His \\
\hline & CCA & Pro & CGA & Arg & CTA & Leu & CAA & Gln \\
\hline & CCG & Pro & CGG & Arg & CTG & Leu & CAG & Gln \\
\hline \multirow{4}{*}{ G } & GCT & Ala & GGT & Gly & GTT & $\mathrm{Val}$ & GAT & Asp \\
\hline & GCC & $\mathrm{Ala}$ & GGC & Gly & GTC & Val & GAC & Asp \\
\hline & GCA & Ala & GGA & Gly & GTA & Val & GAA & Glu \\
\hline & GCG & Ala & GGG & Gly & GTG & $\mathrm{Val}$ & GAG & Glu \\
\hline
\end{tabular}

Red codons-Mixed codons or Syhoms (Synonyms + Homonyms); Blue codons-Synonyms.

If we consider the possible phenomenon of codon VR-transcoding, including synonymous codons, then CFS can be interpreted as a function of the mRNA's semantics (context).

Demonstrativeness of these real material two-way inter-code information exchanges once again suggests that the path of syhom $\rightarrow$ synonymous transcoding can occur virtually, i.e. at the level of their semantics (meanings) for all encoding syhom-synonymous doublets according to the following scheme: one word is written but another word is understood. In general, this real phenomenon can be signifying a warning informing message to us about the importance of understanding the protein code as a dynamic system of meanings, originally set by the texts of genes at the stage of their transcription into mRNA and, secondly, at the stage of their translation into semantic proteins. We have studied such a path as a property of "delegation" of other meanings (semantics) from mRNA to "wobbling" (according to F. Crick) 3'-codons (syhoms) [2]. In other words, if understood generally, semantic (virtual) transcoding of all codons takes placeboth synonyms and syhoms, triggered by mRNA texts. However, we would like to emphasize that syhoms are obvious subjects of transcoding, determined by the context (semantics) of mRNA. This is something that was not noticed by the fathers of the protein code model-F. Crick and M. Nirenberg [3]. This transcoding, that we postulate, does not mean that in such organization of genome operation, there will be a case of a codon semantic chaos. On the contrary, such a genome attribute provides biosystems with broad possibilities to adapt to a volatile external environment. Perhaps, the same phenomenon lies at the basis of Thinking and Consciousness, which are built on the information functions of rapidly synthesized and decaying special linguistic sign (semantic) textual proteins in the cerebral cortex. A highly organized set of their meanings (semantics) 
can represent a semantic field-the equivalent of non-material Thinking and Consciousness with their subsequent materialization in textual proteins. The described VR-transcoding is a manifestation of elementary thinking and consciousness at the genome level. Thinking and consciousness have a distinct fractal structure [4].

The above-mentioned VR-transcoding is a manifestation of elementary thinking and consciousness at the genome level. This is the result of reading and understanding genes (mRNA texts) by a protein synthesizing system. Thinking-consciousness of this level has a hierarchy of fractal dimensions at the stage of mRNA translation.

The smallest of them, the first dimension is represented by the letters (nucleotides) in triplets. The significance of the first dimension is significant: the substitution of each letter, especially the third (wobbling one, according to F. Crick) in these codons, plays a huge role in transforming the meanings of mRNA texts.

The second level of the fractal dimension of the letters of triplets is represented by a family of nucleotide pairs, that is, the first and second codon letters, which in 32 synonymous triplets redundantly but unambiguously encode amino acids. The third letter wobbles, that is, it is not involved or is the object of VR-transcoding.

The third level of the fractal semantic dimension of letters in triplets is represented by the triplets of nucleotides as integral semantic units of 32 codons-synonyms, unambiguously encoding amino acids, already as letters of the semantic continuum of proteins as texts. But they can also be the objects of VR-transcoding. At the same level of fractal dimension, there are 32 syhom codons, that initially ambiguously encode amino acids and stop positions. Syhoms become unambiguous in the process of reading the mRNA context by ribosomes.

All three levels of fractal semantic dimensions contribute a variety of proteins as texts, making semantic continua. The fourth, fifth, and so on levels of codon unification as precursors of words and sentences (texts) follow, but this occurs only at the stage of protein translation. The fourth and subsequent levels of fractality transcend genomes to infinite levels of non-material, mental coding, that is, the creation of programs of conscious behavior and communication of people in verbal-written forms.

In this regard, there is an essential feature of genome fractalization: shifting to higher levels of compression of their semantic dimensions, for example, to the level of arithmetic attributes of the genetic apparatus. In one of his works, analyzing the quantitative ratios of the nucleon composition of the atoms nuclei of encoded amino acids and codons of the triplet genetic code, V.I. shCherbak suggests the presence of an arithmetic calculus in protein biosynthesis, which is also a manifestation of some aspects of the genome quasi-thinking. In the protein code V.I. shCherbak discovered the system of genetic calculus and its use of zero. shCherbak believes that this is an extremely important circumstance, and it is difficult to disagree with him. And here's why: "Zero is a purely intellectual 
and extremely abstract concept, which sets and lays the foundation for co-ordinate thinking and consciousness, making possible quantitative measurements of the external world. These measurements are then interpreted by the internal organismic genetic computing consciousness. So, digits (along with letters) become an integral part of the genetic (protein) code." Therefore, arithmetic control in linguistic (and/or textual) genetics is real, as postulated by V.I. shCherbak.

Developing his ideas, V.I. shCherbak writes: "some cell organelles should work as biocomputers. Thereby, we have to discover the number systems with which they work." And further: "...it seems that the genetic code is connected more closely to abstract notions of arithmetic than with notions of physics or chemistry." [5].

It seems to us that these two positions of shCherbak are not entirely accurate. The chromosomal continuum is already a biocomputer. Probably, it is not self-sufficient and is an integral part of cellular and tissue computing with the use of additional cellular organelles. V.I. shCherbak considers the binary logic of digital computing of the genome to be the determining factor of its operation. He sees the translation of digital DNA-RNA "understanding" into analog form only as a secondary, subordinate path. If this is true, then, only partially. The chromosomal continuum, as a biocomputer, has no strict need to use only the equivalents of wealth (i.e. numbers), it works directly with wealth itself when it is necessary to build an integral organism, and not just synthesize proteins. But binary digital logic is not completely abolished. It is necessary, for example, at the moments of turning on and off protein and RNA genes, which is also important, especially for constructing protein "phrases" and "texts".

At the same time, V.I. shCherbak's research is fundamental, it is of a paradigmic significance, for the first time giving unambiguous mathematical proof that the protein code is a quasi-conscious system and at the same time the result of the semantics of the Universe. The origin of the protein code can only be understood as a conscious act, but not as the result of the blind Darwinian evolution.

Such functioning can be represented as the highest compression of the enormous meaning of the ultimate abstraction of the concept of zero. If applied from this position to the work of the genomes of individual neurons, the level of consciousness-thinking related to them has a relatively small fractal dimension. Here, the highest dimension is probably in the genomic continuum of neurons in the human cerebral cortex. This occurs through the described semantic transcoding of codons with the biosynthesis of short-living semantic proteins, as equivalents of thinking-consciousness. In fact, this is the embodied language of the brain genome. The same can be seen in natural, primarily non-verbal languages. And this corresponds to a more correct translation of the Biblical "From the first he was the Logos" ("In the beginning was the idea/thought"). Thought materialization occurs in speech and in writing. Genomic writing is a textual amino acid sequence in the semantic proteins of the human cerebral cortex. 
Quickly decaying, such proteins can convert into long lasting "so-called" Schrödinger wave holographic memory in the cerebral cortex [6]. One might think that the genetic memory of protein genes is hardware, and VR-transcoding of triplets and the entire mobile organization of protein synthesis is software. In essence, encoding and transcoding, act as the language of the genome. Basically, we can say that the highest genetic code is Thinking-Consciousness in a form of human language, and the Universal language of DNA is a manifestation of it. This can be seen as an antithesis to the abundance of the proposed versions of the "second genetic codes". Their "pandemonium", which Trifonov so sarcastically writes about [7], is only a reflection of VR-transcoding of codons that do not follow the one-dimensional logic of geneticists. Neither second nor third genetic codes exist, including the codes of codes. There is a fractal hierarchy of genetic languages, ascending to the top of human natural languages as a single super-code multi-vector directive, in which the genetic code of proteins is only one of its manifestations.

On the other hand, it's worth remembering the famous phrase attributed to the Bible: "A thought once spoken is a lie." The word is a materialized otherness of a thought, idea. And here comes an eternal question: are thoughts (words) true? For this, let's turn to famous V.V. Nalimov's monograph "The Probabilistic Model of Language", where he proves that any word or their combination in speech and writing always represents a certain field of probabilistic meanings described by the Bayes-Nalimov formula [8]. If the genetic code is the language of the genome, then, its probability is obvious and corresponds to VR-transcoding of triplets as a manifestation of linguistic sign dynamics of genes and their combinations. Any violation can be interpreted as a pathological condition of the biosystem at all levels of the genome-from molecular to organismic and mental. From these positions, the definition of the genetic code of the highest fractal dimension can be refined as a continuum of probabilistic meanings of synthesized proteins during the work of the cerebral cortex [4].

It is the dynamics of thinking and consciousness, that requires the use of an infinitely diverse combinatorics of transcoding of the Code's triplets, resulting in the protein complement as a material reflection of Consciousness-Thinking. To some extent, this is theoretically justified for the wobbling (according to F. Crick) 3'-nucleotide of 32 syhom codons, [1] [2], therefore, we can assume that this possibility is realized on all three nucleotides (letters) of all 64 codons of the Code Table.

The suggested virtual mRNA-context-dependent substitutions of letters (nucleotides) in mRNA codons can be represented as follows during protein biosynthesis:

1) The substitution of the first two nucleotides in the syhom codons with any first two nucleotides from the synonymous codons turns syhoms into any synonymous codons, which is specified by mRNA context.

2) The substitution of the first two nucleotides with any first two letters from other codon families gives the entire table of codon semantics. Moreover, such 
substitutions of the second letters in triplets are hardly noticeable, since they are virtual. They change the meaning of the triplets, physically they remain unchanged.

3) Substitutions of the second letters in codon families provide the same possibilities-that is, they can represent the entire table of codon semantic values. This also occurs virtually, non-materially, depending on mRNA context and thus, cannot be experimentally registered, for example, in PCR systems.

4) The substitution of the 3rd wobbling letter in codons occurs according to the same scenario of ribosomes mRNA-dependent-orientations and plays a key role in switching genome operation to the mental-textual path, which is described in detail in [1].

5) The materialization of virtual substitutions of triplets and their letters occurs during the translation of mRNA into amino acid textual sequences of synthesized proteins, which can be detected by protein sequencing and checking their "amino acids-codons" collinearity. The absence of some collinearity will prove the correctness of this explanation of genome operation.

6) With respect to the 3 '-nucleotide in syhom codons, the following rule can be formulated: Syhoms, in each of their classes, with virtual (or real-mutant, artificial) changes of their own 3'-nucleotides are invariant in meanings programmed by mRNA.

Thus, the verification will provide some basis to believe that the tables of genetic codes of proteins are non-stationary, dynamic and are determined by the meanings of mRNA (and by delegating these meanings to the codons as to integral semantic units and/or the 1st, the 2 nd and the 3 rd nucleotides). The tables of genetic codes can and should be correctly understood only through the dynamics of protein biosynthesis.

The hypothetical-probabilistic nature of this version of genome operation is somewhat balanced by significant experimental results that at first sight are not directly related to transcoding manipulations in the genome. But then, there was an article by F. Crick and M. Nirenberg [3] awarded with the Nobel Prize in 1968, which introduced a universally accepted model of the protein genetic code. The paradox is that this article describes an experiment (we would like to note that it is a key experiment), which was not explained in the part describing operation of the poly U-RNA matrix encoding two different amino acids, phenylalanine and leucine. This fact violates one of the main postulates of the canonical model of the Code proposed by the authors-its unambiguity. The unambiguity of the Code implies that each triplet encodes one and only one amino acid or stop position. F. Crick and M. Nirenberg did not explain this contradiction, saying that the molecular nature of this phenomenon is incomprehensible to them: "An important point to notice is that although the genetic code has certain regularities-in several cases it is the first two bases that encode one amino acid, the nature of the third being irrelevant-its structure otherwise makes no obvious sense." This misunderstanding (which destroys the Code model) had remained unexplained, up to the recent publications [1] [2]. Another experimental 
work, which voluntarily or involuntarily established the confusion in genetics, is an article by Pruit, Lolly and co-authors, which also demonstrated an inexplicable phenomenon [9]. Their study demonstrated that the wild and mutant Hot Head genes of the Arabidopsis thaliana plant, genes with the same DNA sequences, cause different plant biomorphogenesis. This was regarded by some biologists as a deviation from Mendelian genetics. Finally, Turanov et al. published an article [10], which showed the same phenomenon of the simultaneous coding of two different amino acids, cysteine and selenocysteine, by a single codon of UGA in a ciliate (Euplotes crassus infusoria). The authors once again provided an in vivo demonstration of what F. Crick and M. Nirenberg first demonstrated in vitro [3]. All three articles have a strategic, but disguised and misunderstood logical motive, associated with the role of the 3'-nucleotide of syhom codons as a switch of genome Code operation into textual semantic modes, as we mentioned above. In the case of F. Crick's and M. Nirenberg's article, this was about misunderstanding of the function of the UUU syhom codon in a form of poly-U RNA. Its function is mRNA-oriented selection of the desired amino acid-phenylalanine or leucine. But since poly-U RNA has no context, there is no clear choice, therefore, both amino acids are included in the growing peptide, which clearly violates the principle of the Code model unambiguity.

In [9], the situation is different. The authors of Lolly et al. encountered a phenomenon that seems to contradict classical Mendelian genetics. In the case of an excellent study of the genetic code of the ciliate Euplotes [10], the situation is even more complicated. The genetic apparatus of infusoria has the same task: having one triplet of UGA in the mRNA, which encodes cysteine and selenocysteine at the same time, it is obliged to choose only one from two different amino acids. This occurs due an additional RNA fragment insertion into the mRNA - a short hairpin palindrome. It is the RNA palindrome that changes the context of mRNA, which determines the selection of one amino acid from two different ones. The semantic orientation of the ribosome in the context of mRNA was used to select the desired amino acid. This demonstrates that a hairpin palindrome can also serve in another capacity-as a topological linguistic sign structure with a function of signaling the selection by the ribosome of one of two different amino acids.

Explanations of these paradoxical and incomprehensible purely experimental results lie somewhat separately from classical genetics. These concepts were formulated in [1] [2], including the key concept of ambiguous coding of amino acids due to the second, homonymous, degeneracy vector of the protein code. In 1997 this was predicted by P. Gariaev in [11].

This phenomenon is about the twofold synonymous-homonymous degeneracy of the protein Code. This automatically stems from F. Crick's postulate on the wobbling of the third nucleotide ( 3 ' nucleotide) in the syhom codons. As described above, wobbling is virtual at the stage of mRNA transcription and materializes into reality at the stage of mRNA translation into proteins [1] [2]. In another words, it can be formulated as follows: the genome operates in three 
strategic dimensions-material, linguistic and quantum.

1) The physical/material dimension is the real genome: chromosomal continuum and DNA.

2) The linguistic dimension is the textual-semantic content of informational biomacromolecules-DNA (genes), mRNA (transcriptional, textual representation of genes) and Proteins (translational, protein-textual representation of genes). These are three textual dialects (DNA-RNA-Protein), giving the same information.

3) The quantum dimension is the non-material, wave representation of DNARNA-Proteins. For example, in the form of physical torsion fields or spintronic effects of transmission of active quantum equivalents of genes at macro distances [12]. This is an ideal (from the word "idea") display of the triad of DNA-RNAProtein semantics. The quantum dimension has an additional and essential hypothetical attribute-nonlocality. The nonlocality of genetic information at the quantum level is divided into two more sublevels a) holographic nonlocality; b) nonlocality within the framework of Einstein-Podolsky-Rosen phenomenon (EPR).

The analyzed works [3] [9] [10] contradict the F. Crick-M. Nirenberg Code model not tactically, but strategically, since they explain and prove the role and significance of the second synonymous-homonymous vector of the Code degeneracy. The second vector of genome operation transcends biosystems to endless horizons for using genes and their protein products as textual, semantic structures.

What objections can be raised to the basic idea of this article on the virtual-real transformations of codon meanings during protein biosynthesis, transformations that depend on the contexts (meanings) of mRNA? The first and main objection is massive total violations of thermodynamically substantiated rules for pairing of codon-anticodon pairs: A-U, G-C. Though in his work on the Wobble Hypothesis [13], F. Crick gave examples of such violations: the use of inosine in tRNA anticodon. By extrapolating this violation to all codon-anticodon recognitions and focusing on the explanation of virtual-real transcoding of codons, we can say the following. The biological (informational) value of violations of the A-U, G-C rule is significantly higher than the thermodynamic troubles arising from this.

Other objections may be related to the problems of mutual recognition of tRNA and aminoacyl-tRNA synthetases, which are forced to adapt to the emerging pseudo chaos with the changed rules of codon-anticodon pairings. But such adaptation is inevitable, otherwise it may result in real semantic chaos. There is also a positive point within these problems. Biosystems use seemingly unthinkable huge amounts of tRNA, varying in different species. It is logical to think that their numbers depend on the mobile semantic realms of genes' meanings, placed in different contexts of genes due to their transpositions. The latter can be considered as a way to realize new gene meanings. And such cases are known: for example, the transition of "silent" genes to "speaking" ones or a 
precedent of changing the semantics of the Hot Head gene, which does not change its sequence, but changes its morphogenetic functions [9]. These are examples of the famous so-called "Gene position effect". And this is the same phenomenon of virtualization-realization of codon meanings, which depends on the contextual meaning of mRNA sequences and genes. All this sets a different vector for working with DNA-mRNA-protein sequences. And only now, this vector is beginning to organize our work differently.

\section{Discussion}

Genetics and molecular biology abruptly transcend to a new level of understanding of genome operation. This transcendence is strategically determined by the real textual attributes of the genome, which we have been trying to understand for a long time as a nanobiocomputer operating in two main modes: 1) real (non-metaphorically) textual and 2) quantum. If we buy this idea, many current problems of genetic coding will appear in a clear and pragmatic form. And this means an opportunity to put genetics, medicine, agriculture and quantum computing on a different stable foundation. The linguistic nature, the real textual nature of the genome, is the subject of another consideration, begins with the Wobble Hypothesis of F. Crick, who did not realize its consequences, being unable to understand its substantially deeper biological meaning [14] [15] [16]. What is this meaning about?

Let's turn to the book of F. Crick' memoirs. In this book he writes in relation to his Wobble hypothesis something more substantial than in all his previous articles: "An important point to notice is that although the genetic code has certain regularities-in several cases it is the first two bases that encode one amino acid, the nature of the third being irrelevant-its structure otherwise makes no obvious sense."

The meaning of this phrase requires close analysis if we want to understand the coding of proteins at the level of DNA with respect to the 3'-nitrogen base in the syhom codon in the $3^{\prime}-5$ 'codon-ancodon pair. The meaning of this phrase is not unambiguous. This refers primarily to the words "...the nature of the third being irrelevant-its structure otherwise makes no obvious sense." That is, if you take the "otherwise" case as the truth, the meaning and purpose of the code becomes not obvious. But (!) F. Crick does not use the word "incorrect" or "meaningless" structure of the genetic code. He said, "it is not obvious". And the second uncertainty in these citations from F. Crick is "in several cases, it is the first two bases that encode one amino acid". By the time the book was written, it was already known that this was not only several cases, but the function of 32 codon-synonyms. The second half-32 syhom-codons remained for F. Crick in the shadow of uncertainty. As, by the way, it remains until now for many molecular biologists and geneticists. It is here, where the general misunderstanding of the role of syhom-codons and their "wobbling" 3'-nucleotides is hidden and obscured. And the meaning that F. Crick invested into the word "wobbling" also remains unclear. Are these the substitutions of 3'-nucleotides? But such substi- 
tutions can only occur due to mutations that normally do not exist. Or is it a substitution of semantic values of 3'-nucleotides in syhom-codons (as we believe)? So that these are not the third nucleotides in the syhoms that wobble, but these are the meanings of the 3 '-nucleotides in the codons that virtually wobble. And this wobbling is not physical, but "mental", dependent on the meaning of mRNA. The meaning wobbles, similar to how in a spoken language when incorrectly pronounced letters wobble within words-we mentally correct them, knowing the general meaning of the pronounced phrase. This is what the "no obvious sense" meaning of the presence of the third wobbling nucleotide in 32 non-synonymous codons (syhoms), is about: the coding value of this 3rd nucleotide is delegated by the mRNA context, as an ideal construct (from the word "idea") [2]. This is precisely what F. Crick and M. Nirenberg did not understand when they received a brilliant experimental result of encoding by the UUU syhom-codon of two different amino acids-phenylalanine and leucine. Inside the Nobel's model of M. Nirenberg's genetic code, perhaps, there was one more Nobel Prize. But they said that "The molecular basis of this ambiguity is not known. Nor is it known if the dual coding occurs in living systems as well as in cell free systems" [3]. It was in this fundamental discovery where the explanation of the speech-likeness of protein genes was hidden [1] [2]. And at the same time, it was referring to the mental, conscious nature of protein genetic coding, since the choice of two different amino acids simultaneously encoded by the syhom-codon can only be made after reading and understanding the meaningful context of the mRNA text using the ribosome as a nanobiocomputer. This was understood only in the following studies [2] [11]. The wobbling of the 3'-nucleotides of syhom-codons (hybrids of synonyms and homonyms) is a strategic switch of the protein code to the text mode of operation. The key point of such switching is found in the fact of virtual delegation of new semantic values to the 3'-nucleotides of syhom-codons. These meanings are set by the contextual (semantic, non-material) contents of the given mRNAs during their transcriptions [1] [2] and are materialized at the stage of their translation into selected in this way amino acids and/or protein biosynthesis is stopped. Here, the most subtle and significant factor is the context-sensitive-mRNA-dependent quasi-conscious choice of one amino acid from two different ones with undetermined double coding of them by syhom-codons. Or the choice of the "stop meaning" of the syhom-codon. De facto, the second syhom-degeneracy of the protein code was experimentally proved in the methodically brilliant work of Turanov et al. [10]. But they failed to point out the value of this phenomenon. In this predominantly theoretical study, we attempt to develop the idea of a larger significance of $\mathrm{F}$. Crick's original thoughts expressed by him in [13] regarding the wobbling 3'nucleotide in the syhom-codons.

\section{Conclusion}

Francis Crick introduced the Wobble Hypothesis of 3'-nucleotides of codons of the genetic code of proteins, which logically leads to the idea of its double syn- 
onymous-homonymous degeneracy. This leads to large consequences not seen by F. Crick:

1) The terms "syhom" and "syhomy", introduced by us, reflect the twofold degeneracy of the protein code-by the vector of synonymy of the first half (32 codons) and by the vector of homonymy of the second half ( 32 codons).

2) Synonymy and syhomy of codons is a condition of multiple redundancy and accuracy (unambiguity) of the coding of amino acids and stop positions. In synonymy, the redundancy and accuracy of amino acid coding is ensured by the presence of iso-acceptor transport RNAs. In homonymy, the redundancy and accuracy of coding of amino acids and stop positions is ensured both by wobbling of the 3'-nucleotides of all three positions in triplets of codons-syhoms, leading to their virtual-real transcoding, and their virtual-real transcoding at the level of values of syhom-codons triplets as integral semantic units.

3) Homonymy and at the same time synonymy in mixed 32 syhom codons is realized within pairs and between pairs of triplets in each of the 8 families of syhom codons. This division makes it necessary to SELECT one amino acid from two different amino acids when a ribosome meets syhoms. The choice is made (attention!) according to the MEANING of the mRNA contexts. This is a huge evolutionary leap of the Code, indicating the MENTAL basis for coding proteins along the syhomy vector. Ribosomes have to choose from multiple aminoacylated tRNA pairs that carry different amino acids and/or stop command. This is the greatest evolutionary achievement of the protein Code. This choice is based on quasi-conscious acts of understanding of meanings of mRNA-protein-synthesizing-system as a nanobiocomputer.

4) All described highly organized acts begin with VIRTUAL (Crick's) wobbling of the 3'-nucleotide in the mRNA's syhoms, triggering the uncertainty, duality of coding of amino acids and stops. Why is wobbling virtual? It is semantic, defined (programmed) by the meanings of mRNA, affecting the semantic transcription of mRNA. This is an analogue of a mental (virtual) correction by us of someone's burry speech who does not correctly pronounce some letters or words. It is this ingenious simplicity that is used by ribosomes as part of the entire protein synthesizing system with the functions of nanobiocomputing.

5) Virtuality (non-materiality of mRNA meanings) disappears when mRNA transcripts are translated into real amino acid sequences of proteins. The meanings of genes in their speech (mental) aggregates materialize into material amino acids.

The main, summarizing consequence of the given positions is as follows:

Syhoms, in each of their classes, with virtual (or real-mutant, artificial) changes in their 3' nucleotides, are invariant in meaning defined (programmed) by $m R N A$. How can we experimentally verify the key idea of semantic invariance of values of 3' nucleotides of codon-syhoms and codon-syhoms as integral semantic units?

If you follow traditional genetics, then, any artificial damage of syhom-codons-mutations, nucleotide substitutions in all three positions in the active 
centers of enzyme genes or in other bioactive proteins, will lead to their inactivation. Transcending the framework of traditional genetics with proposed phenomenon of syhomy, allows us to predict that there will be no inactivation of enzymes or other bioactive protein substances. This is prevented by the semantic content of mRNA texts, which correct distortions of syhom-codons.

\section{Conflicts of Interest}

The authors declare no conflicts of interest regarding the publication of this paper.

\section{References}

[1] Gariaev, P.P. (2015) Another Understanding of the Model of Genetic Code. Theoretical Analysis. Open Journal of Genetics, 5, 92-109. https://doi.org/10.4236/ojgen.2015.52008

[2] Gariaev, P.P. and Leonova-Gariaeva, E.A. (2018) The Syhomy of the Genetic Code Is the Path to the Real Speech Characteristics of the Encoded Proteins. Open Journal of Genetics, 8, 23-33. https://doi.org/10.4236/ojgen.2018.82003

[3] Crick, F.H. and Nierenberg Marshall, W. (1962) The Genetic Code, I II. Scientific American, 207, 66. https://doi.org/10.1038/scientificamerican0363-80

[4] Gariaev, P.P. (2014) Fractality of the Intellect. (In Russian) https://ss69100.livejournal.com

[5] Cherbak, V.I. (2003) Arithmetic inside the Universal Genetic Code. Biosystems, 70, 187-209. https://doi.org/10.1016/S0303-2647(03)00066-2

[6] Nobili, R. (1985) Schrödinger Wave Holography in Brain Cortex. Physical Review $A$, 32, 3618-3626. https://doi.org/10.1103/PhysRevA.32.3618

[7] Trifonov, E. (2012) Pandemonium of the Second Genetic Codes. Troitsky Variant, 95, 13. (In Russian) https://trv-science.ru/2012/01/17/stolpotvorenie-vtorykh-geneticheskikh-kodov

[8] Nalimov, V.V. (1979) A Probabilistic Model of Language. Monograph, The Science, Moscow, 303. (In Russian)

[9] Lolle, S.J., Victor, J.L., Young, J.M. and Pruitt, R.E. (2005) Genome-Wide NonMendelian Inheritance of Extra-Genomic Information in Arabidopsis. Nature, 434, 505-509. https://doi.org/10.1038/nature03380

[10] Turanov, A.A., et al. (2009) Genetic Code Supports Targeted Insertion of Two Amino Acids by One Codon. Science, 323, 259. https://doi.org/10.1126/science.1164748

[11] Gariaev, P.P. (1997) Wave Genetic Code. Publishing Center, Moscow, 107. (In Russian)

[12] Gariaev, P.P., Kokaya, A.A., Mukhina, I.V., Leonova-Gariaeva, E.A. and Kokaya, N.G. (2007) Effect of Electromagnetic Radiation Modulated by Biostructures on the Course of Alloxan Diabetes Mellitus in Rats. Bulletin of Experimental Biology and Medicine, 2, 155-158. (In Russian) https://doi.org/10.1007/s10517-007-0049-3

[13] Crick, F.H. (1966) Codon-Anticodon Pairing: The Wobble Hypothesis. Journal of Molecular Biology, 19, 548-555. https://doi.org/10.1016/S0022-2836(66)80022-0

[14] Shipov, G.I. and Gariaev, P.P. (2018) Quantum Genome in Terms of the Physical Vacuum. "Conceptual". Publishing House, Moscow, 152. (In Russian)

[15] Gariaev, P.P. (2019) Quantum Consciousness of the Linguistic-Wave Genome. 
Theory and Practice. Institute of Linguistic/Wave Genetics. (In Process of Publication)

[16] Gariaev, P.P. (2019) Linguistic-Probabilistic and Quantum Understanding of Gene Operation. Open Journal of Genetics, 9, 43-64.

https://doi.org/10.4236/ojgen.2019.93004 